\title{
Endogenous Endophthalmitis with Resultant Visual Loss Secondary to Biliary Sepsis
}

Mutsuki Marukawa, Richard P Stevenson, Wan Hei Chan* and Vivien Gough

Department of General Surgery, Royal Alexandra Hospital, UK

*Corresponding author: Wan Hei Chan, Department of NHS Greater Glasgow \& Clyde, UK

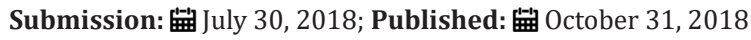

\section{Case Report}

A 63-year-old gentleman initially presented with abdominal pain and jaundice. Ultrasound scan demonstrated gallbladder sludge with common bile duct and intra-hepatic biliary duct dilatation. Subsequent abdominal CT scan demonstrated an obstructed distal common bile duct of unclear etiology. Endoscopic retrograde

cholangiopancreatography (ERCP) confirmed a malignant stricture resistant to cannulation with brush cytology indicating a cholangiocarcinoma. Subsequent percutaneous transhepatic cholangiogram (PTC) with stent insertion decompressed the biliary system.

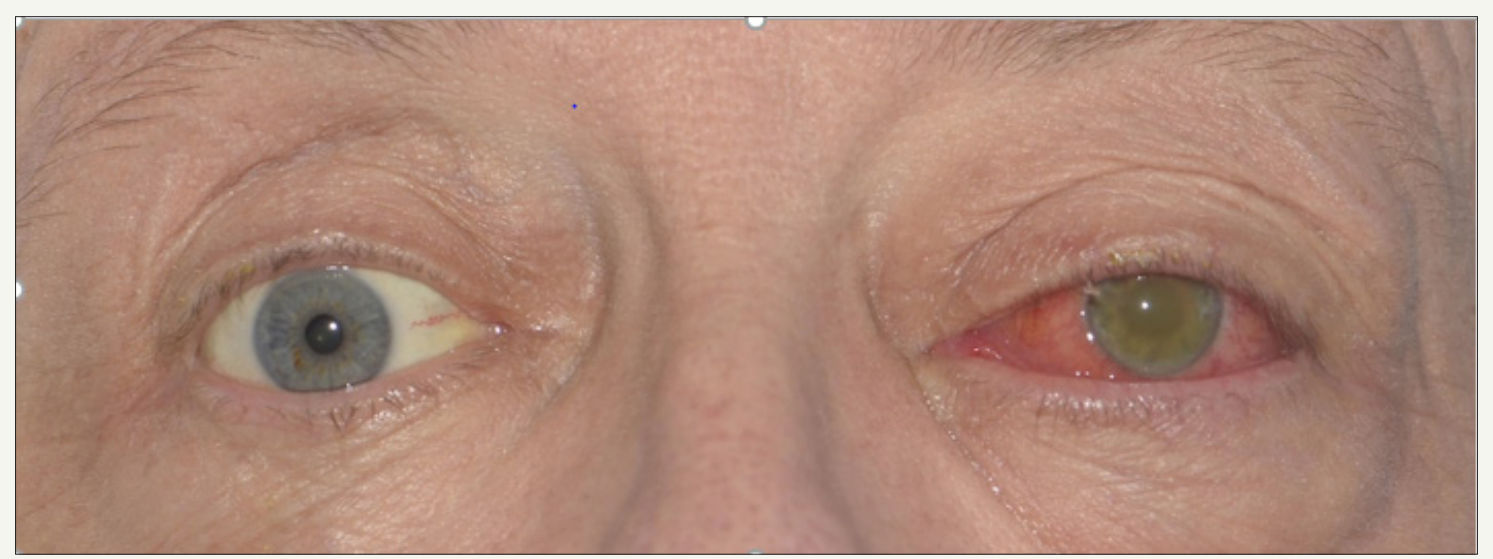

Figure 1: Left eye. Note erythema in conjunctiva and sclera with haziness in anterior chamber. According to the patient, this had developed over the course of a day.

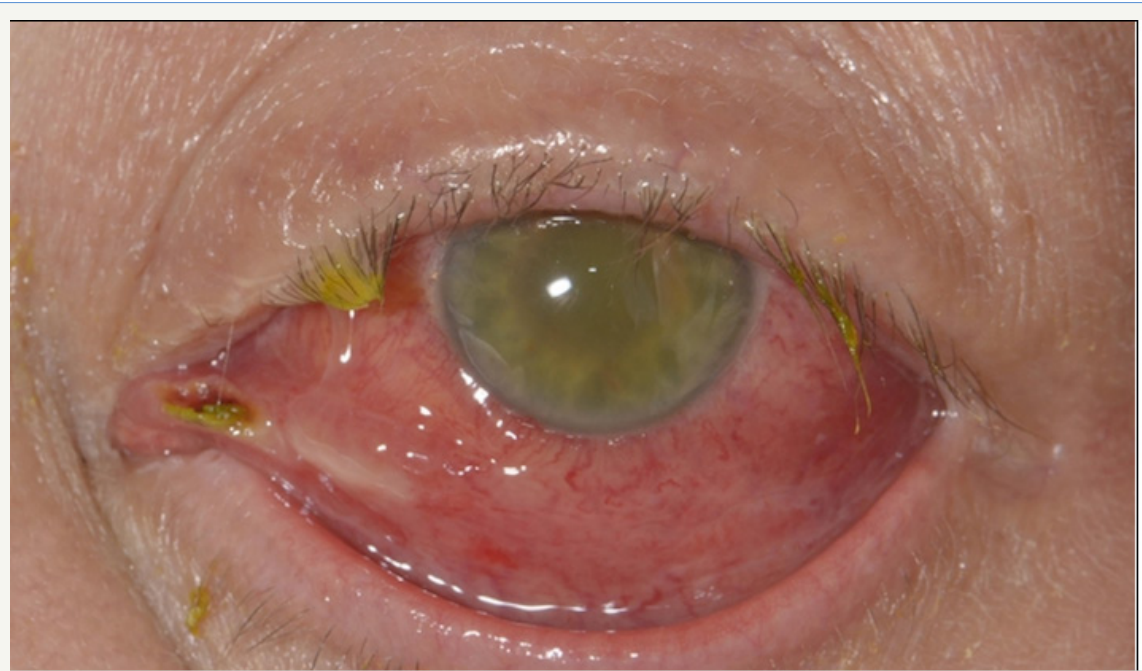

Figure 2: Image comparing eyes - ptosis and peri-orbital soft tissue swelling is more evident in this photo, with pupils not as reactive to light. 
1 week later following discharge he re-presented with acute visual loss affecting his left eye with concurrent biliary sepsis. His left eye had light perception only, proptosis and raised intraocular pressure (Figure 1). His right eye was unaffected with a visual acuity of 6/6 (Figure 2). CT head showed orbital swelling and three sets of blood cultures were negative for organisms. He underwent a virtual biopsy that grew Escherichia Coli. He was treated empirically with intravitreal, topical and intravenous antibiotics for endogenous bacterial endophthalmitis secondary to ascending cholangitis. Despite aggressive treatment his left eye remained nonperceptive to light with a dense fibrin plaque noted to be covering the pupil with posterior synechiae and no fundal view. Ultrasound demonstrated no evidence of retinal detachment.

He was initially planned for total pancreatectomy and splenectomy, however subsequent mesenteric biopsies taken at staging laparoscopy confirmed metastatic disease and as such he was commenced on Gemcitabine-Abraxane palliative chemotherapy.

Endogenous Endophthalmitis (EE) is a rare and debilitating infection of the eye whereby the causative organism is spread hematogenous from an extra-orbital source of infection. Most patients have one or more risk factors which include malignancy, intravenous drug use, diabetes mellitus, hepatobiliary disease or indwelling catheters [1]. Causative organisms are either fungal or bacterial. In the West/Europe Gram positive bacteria (Streptococcus and Staphylococcus) from endocarditis or soft tissue infections predominate, whereas in East Asia Gram-negative Klebsiella are the commonest. EE caused by Gram-negative bacteria are rare in the West/Europe [2].

Management principles include a full septic screen with vitreous aspiration. In one study vitreous aspirates were positive on $87 \%$ of presumed EE cases but blood cultures were only positive in 33\% [3]. Prognosis is better with early vitrectomy along with systemic and local antibiotics [3].

Outcomes following EE are poor; in one study 33\% of patients developed ocular complications (other than decreased visual acuity) and 54\% had visual acuity worse than 20/400 with many of these cases requiring enucleation/evisceration [3]. Better outcomes are associated with early diagnosis and treatment, but this is difficult as there are more common causes of sudden visual acuity loss compared to EE.

\section{Learning Points/Take Home Message}

A. Endogenous endophthalmitis is a rare but debilitating disease which is more common in patients with known risk factors. Due to haematogenous spread, a high index of suspicion is required when a patient presents with visual acuity loss following/ concurrent with a severe systemic infection, particularly if they have known risk factors.

Early intervention and detection of the causative organism is the key to preserving visual acuity in these patients.

\section{References}

1. Scheidler V, Scott IU, Flynn HW, Davis JL, Benz MS, et al. (2004) Cultureproven endogenous endophthalmitis: Clinical features and visual acuity outcomes. Am J Ophthalmol 137: 725-731.

2. Wong JS, Chan TK, Lee HM, Chee SP (2000) Endogenous bacterial endophthalmitis; An East Asian experience and a reappraisal of a severe ocular affliction. Ophthalmology 107: 1483-1491.

3. Ness T, Pelz K, Hansen LL (2007) Endogenous endophthalmitis: Microorganisms, disposition and prognosis. Acta Ophthalmol Scand 85(8): 852-856.
Creative Commons Attribution 4.0 International License

For possible submissions Click Here

\section{Submit Article}

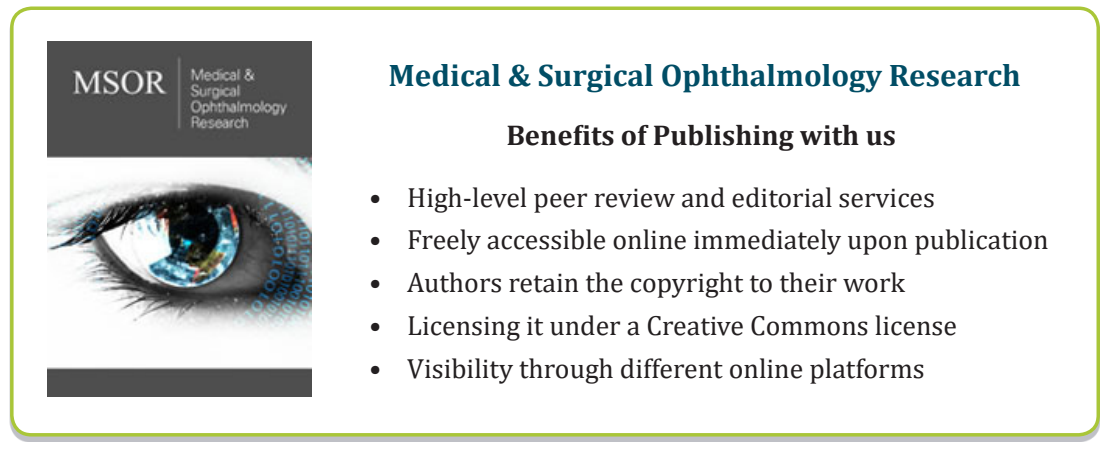

